\title{
Role of nanoscale antigen organization on B-cell activation probed using DNA origami
}

\author{
Rémi Veneziano 1010,11, Tyson J. Moyer 2,11, Matthew B. Stone ${ }^{1,11}$, Eike-Christian Wamhoff',

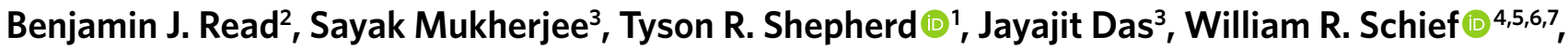 \\ Darrell J. Irvine $\mathbb{B}^{1,2,3,6,7,8,9 凶}$ and Mark Bathe $\mathbb{B}^{1 \bowtie}$
}

\begin{abstract}
Vaccine efficacy can be increased by arraying immunogens in multivalent form on virus-like nanoparticles to enhance B-cell activation. However, the effects of antigen copy number, spacing and affinity, as well as the dimensionality and rigidity of scaffold presentation on B-cell activation remain poorly understood. Here, we display the clinical vaccine immunogen eOD-GT8, an engineered outer domain of the HIV-1 glycoprotein-120, on DNA origami nanoparticles to systematically interrogate the impact of these nanoscale parameters on B-cell activation in vitro. We find that B-cell signalling is maximized by as few as five antigens maximally spaced on the surface of a 40-nm viral-like nanoparticle. Increasing antigen spacing up to $\sim 25-30 \mathrm{~nm}$ monotonically increases B-cell receptor activation. Moreover, scaffold rigidity is essential for robust B-cell triggering. These results reveal molecular vaccine design principles that may be used to drive functional B-cell responses.
\end{abstract}

E fficient activation of antigen-specific B cells is a central goal in the design of new vaccines. One well-established strategy to enhance B-cell activation employs multivalent presentation of immunogens $s^{1}$. Antigen multimers, antigen-conjugated polymers and virus-like nanoparticles (NPs) displaying immunogens at high density have all been shown to strongly initiate early $\mathrm{B}$-cell signalling ${ }^{2-6}$. Antigen multimerization enables low-affinity B cells to be fully activated ${ }^{7,8}$, which may be particularly important for approaches such as lineage-guided or germline-targeting (GT) vaccines that aim to activate specific rare, low-affinity target B-cell populations $s^{9-13}$. However, the independent roles of antigen spatial arrangement and additional design parameters (including antigen copy number, affinity and the rigidity and dimensionality of scaffold presentation) in triggering $\mathrm{B}$ cells and initiating robust B-cell receptor (BCR) signalling remain poorly understood. So far, studies exploring the effect of antigen organization on B-cell triggering have generally employed protein, polymer or particle scaffolds that only allowed limited variation of the preceding design parameters or provided only statistical control over the numbers and locations of antigens $s^{2,3,14-16}$. Here, to independently probe the relative roles of immunogen valency and spacing on immunoglobulin-M (IgM)-BCR activation, we used scaffolded DNA origami NPs ${ }^{17-19}$ to display discrete antigen copy numbers with controlled inter-antigen spacings on the scale of an individual virus-like NP. As a clinically relevant model antigen, we focused on the GT engineered outer domain (eOD) of the HIV-1 envelope glycoprotein antigen gp120, termed eOD-GT8 ${ }^{20}$. This antigen was designed to bind with high affinity to the inferred germline precursor of the VRC01 CD4 binding site-specific HIV broadly neutralizing antibody (glVRC01), and thereby activate a collection of human naive B cells expressing so-called VRC01-class IgM-BCRs ${ }^{13,21,22}$. eOD-GT8 activates both cognate glVRC01 IgM-BCR-expressing cell lines and murine BCR-transgenic primary B cells $\left(K_{\mathrm{D}} \approx 30 \mathrm{pM}\right)$, but only when presented to $\mathrm{B}$ cells in multivalent form ${ }^{8,21}$. Schief and colleagues showed that multimerization of 60 copies of eOD-GT8 via fusion to the self-assembling bacterial protein lumazine synthase formed an isotropic 30-nm-diameter NP (eOD-60mer) that elicited robust B-cell activation in vitro and in vivo ${ }^{12,20,21}$. Here, to interrogate the relative roles of antigen spacing, copy number and the dimensionality and rigidity of scaffold presentation on B-cell activation in vitro, we used DNA origami to spatially present eOD-GT8. Key findings were recapitulated with a lower-affinity eOD-GT variant and model peptide antigens recognized by primary murine $\mathrm{B}$ cells. We also interpret our results in the broader context of a mechanistic, molecular-level reaction-diffusion model of membrane-proximal IgM-BCR signalling kinetics.

To systematically probe the independent roles of antigen copy number versus spacing on B-cell triggering, we focused on two structured DNA-NP ${ }^{17,18}$ variants: a three-dimensional (3D) icosahedral $\mathrm{NP}^{18}$ with a $\sim 40$-nm-diameter size that is comparable to the eOD-60mer and a 1D rigid-rod six-helix bundle (6HB) with maximal dimensions of $\sim 80 \mathrm{~nm}$ (Fig. 1a and Supplementary Figs. 1 and 2). eOD-GT8 antigens were site-specifically coupled to these nanostructures through hybridization to free single-stranded DNA (ssDNA) overhang strands displayed from the origami at specific, spatially programmed locations (Supplementary Fig. 3). Using this approach, we were able to present the antigen in copy numbers varying from 1 to 60 in variable nanoscale spatial

'Massachusetts Institute of Technology, Department of Biological Engineering, Cambridge, MA, USA. ${ }^{2}$ Massachusetts Institute of Technology, Koch Institute for Integrative Cancer Research, Cambridge, MA, USA. ${ }^{3}$ The Ohio State University, Department of Pediatrics, Battelle Center for Mathematical Medicine, The Research Institute at Nationwide Children's Hospital, Columbus, OH, USA. ${ }^{4}$ Center for HIV/AIDS Vaccine Immunology and Immunogen Discovery, The Scripps Research Institute, La Jolla, CA, USA. International AIDS Vaccine Initiative Neutralizing Antibody Center, The Scripps Research Institute, La Jolla, CA, USA. ${ }^{6}$ Department of Immunology and Microbial Science, The Scripps Research Institute, La Jolla, CA, USA. ${ }^{7}$ Ragon Institute of Massachusetts General Hospital, Massachusetts Institute of Technology and Harvard University, Cambridge, MA, USA. ${ }^{\circ}$ Massachusetts Institute of Technology, Department of Materials Science and Engineering, Cambridge, MA, USA. ${ }^{9}$ Howard Hughes Medical Institute, Chevy Chase, MD, USA. ${ }^{10}$ Present address: George Mason University, Volgenau School of Engineering, Department of Bioengineering, Fairfax, VA, USA. "These authors contributed equally: Rémi Veneziano, Tyson J. Moyer, Matthew B. Stone.凶e-mail: djirvine@mit.edu; mark.bathe@mit.edu 


\section{a}

(i)

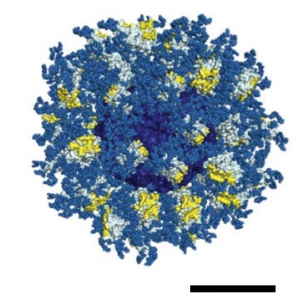

(ii)

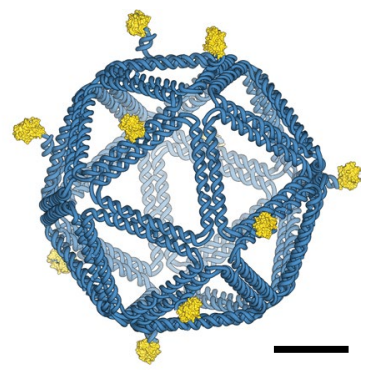

(iii)

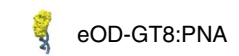

eOD-GT8:PNA

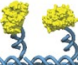

5 copies

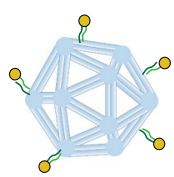

10 copies

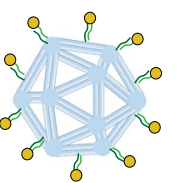

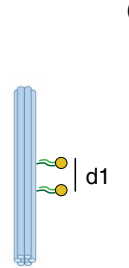

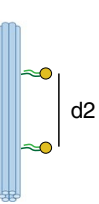

(iii)

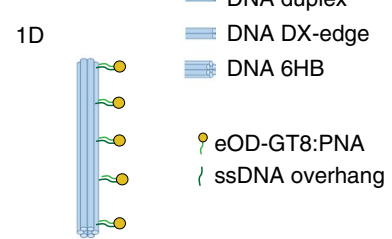

3D

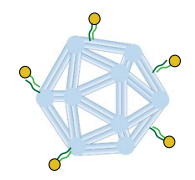

Fig. 1 | Scaffolded DNA origami NPs to control nanoscale organization of HIV immunogens. a, DNA-NPs were designed to self-assemble the eOD-GT8 antigen in a controlled manner, mimicking features of the eOD-GT8-60mer immunogen: (i) eOD-GT8-60mer protein NP; (ii) icosahedral DNA-NP presenting 10 copies of eOD-GT8 (Ico-10X); (iii) $6 \mathrm{HB}$ rod-like structure presenting two copies of eOD-GT8 (6HB-2x). Scale bars, 10 nm. b, Both the icosahedral and $6 \mathrm{HB}$ structures were used to explore (i) the stoichiometry; (ii) the inter-antigen distance, d1 and d2; (iii) the 1D versus 3D dimensionality of eOD-GT8 antigen presentation (DX, double-crossover).

organizations, enabling independent control over antigen stoichiometry, inter-antigen distance, as well as the spatial dimensionality of antigen presentation (Fig. 1b).

As comparative controls to test the impact of DNA-NP scaffold rigidity on IgM-BCR activation, we prepared dimer constructs presented on flexible ssDNA or polyethylene glycol (PEG) polymer linkers of varying lengths (Supplementary Table 1). To generalize our results to other antigens and immunogen-presentation scaffolds, we also examined a lower-affinity variant of eOD-GT8, as well as distinct peptide model antigens, which we displayed using either liposomes or a pentagonal bipyramid origami NP (see Supplementary Information).

Negative-stain transmission electron microscopy (TEM) imaging and agarose gel electrophoresis of the $6 \mathrm{HB}$ and icosahedral DNA-NP constructs confirmed their geometry, monodispersity and structural rigidity, consistent with previous work ${ }^{18}$ (Fig. 2a and Supplementary Figs. 4-7). Short, outward-facing ssDNA overhangs were used at the $3^{\prime}$ ends of select DNA-NP staple strands to anchor individual eOD-GT8 monomers (Supplementary Fig. 3) via hybridization to a complementary peptide nucleic acid (PNA) tag site-specifically conjugated to the antigen (Supplementary Figs. 8-10). PNA strands are synthetic polymers that mimic nucleic acids in their hybridization affinity and specificity via Watson-Crick base-pairing, yet their uncharged peptide backbone, which replaces the phosphate backbone of DNA, results in a higher binding affinity with DNA compared with DNA:DNA hybridization ${ }^{23}$. eOD-GT8-PNA monomers were purified and added to pre-folded and purified DNA-NPs to allow for complete hybridization prior to purification and application to B cells in vitro. Antigen-coupled NPs showed expected shifts in gel electrophoresis (Fig. 2b), while fluorimetry (using a fluorescent version of the PNA-eOD-GT8 labelled with AlexaFluor647 (AF647)) and tryptophan fluorescence measurements confirmed efficient and stable complexation of eOD-GT8-PNA to DNA-NPs with quantitatively controlled stoichiometries varying from 2 to 60 (Fig. 2b and Supplementary Figs. 11-13).

\section{Effect of immunogen organization on B-cell triggering}

We first analysed the impact of eOD-GT8 valency on B-cell response using the $\sim 40$-nm-diameter icosahedral DNA-NP bearing zero to ten copies of eOD-GT8 monomers distributed equidistantly from one another over the surface of the NP ([0-10]-mer, Supplementary Fig. 14 and Supplementary Table 2). As a readout of IgM-BCR triggering, we focused on dynamic measurements of intracellular calcium signalling as a critical signature of full IgM-BCR activation ${ }^{24}$. Human Ramos B cells stably expressing the eOD-GT8 antigen's cognate germline VRC01 IgM receptor were incubated with DNANPs equivalent to $0.5 \mathrm{nM}$ or $5 \mathrm{nM}$ total eOD-GT8, or the same eOD-equivalent concentration of eOD-GT8-60mer protein NPs, with B-cell responses recorded spectroscopically using a fluorescent intracellular calcium indicator dye. As expected ${ }^{8,21}$, antigen-free DNA-NPs or NPs bearing a single copy of eOD failed to activate $\mathrm{B}$ cells at either antigen concentration. In contrast, NPs bearing two or more copies of eOD-GT8 triggered monotonically increasing cellular responses with increasing antigen valency (Fig. 2c,d). Intriguingly, at both antigen concentrations, signalling plateaued with 5-mer or higher valency DNA-NPs, at a level indistinguishable from the eOD-GT8-60mer. Moreover, increasing the valency of the DNA icosahedron to 30 or 60 copies of eOD-GT8 had no further effect on increasing the cellular response (Supplementary Fig. 15). Because the affinity of eOD-GT8 for glVRC01 is high, with a $K_{\mathrm{D}} \approx 30 \mathrm{pM}$ (ref. ${ }^{21}$ ), we also tested the same icosahedral DNA-NP functionalized with a lower-affinity variant, eOD-GT5 $\left(K_{\mathrm{D}} \approx 0.5 \mu \mathrm{M}\right.$; refs. ${ }^{8,21}$ ) (Supplementary Fig. 16). As observed with eOD-GT8, the five- and ten-copy-number icosahedral NPs exhibited a similar plateau in signalling when conjugated with eOD-GT5. Interestingly, however, even at an eOD-GT5 concentration of $25 \mathrm{nM}$, maximum activation remained lower than that observed with the eOD-GT860 mer at the considerably lower immunogen concentration of $2 \mathrm{nM}$.

We next sought to define the impact of antigen spacing on IgM-BCR triggering. Previous studies have shown that inter-antigen separations impact receptor signalling for both Fc receptor engaging $\operatorname{IgE}^{25}$ and the T-cell receptor ${ }^{26}$, and that nanoscale organization of the BCR crucially impacts receptor activation ${ }^{5,27-30}$. To evaluate antigen spacing in the absence of potentially confounding $3 \mathrm{D}$ geometric variation, we turned to a $1 \mathrm{D}$ rigid $\mathrm{DNA}$ origami rod made from a simple $6 \mathrm{HB}$ to first present antigen dimers using variable, fixed inter-antigen spacings (Supplementary Figs. 2 and 3). Calcium signalling in responding $\mathrm{B}$ cells was triggered at the closest dimer 
a

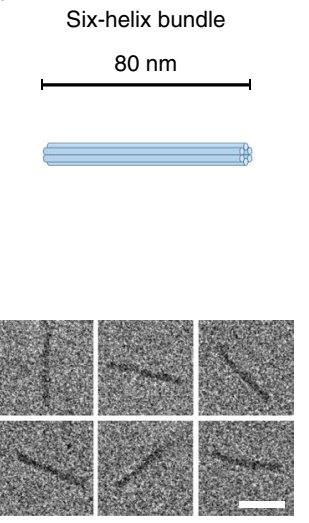

Icosahedron
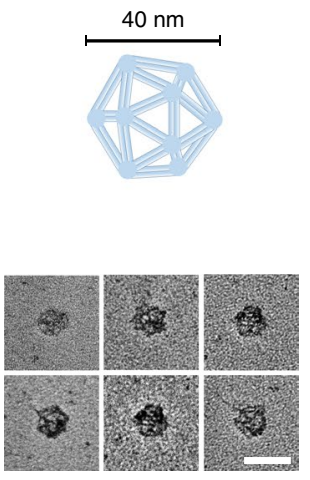

b
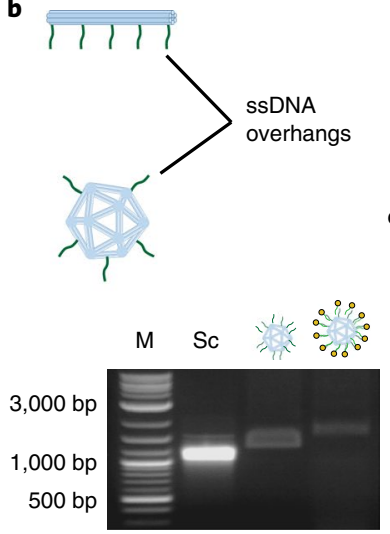

$\longdiv { 8 5 8 5 8 }$
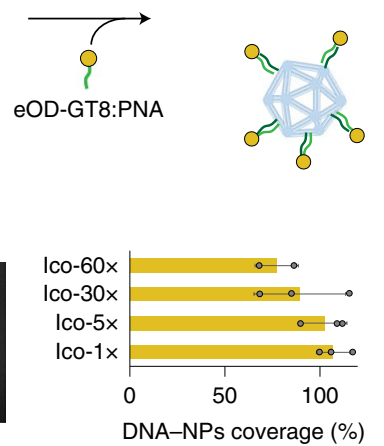

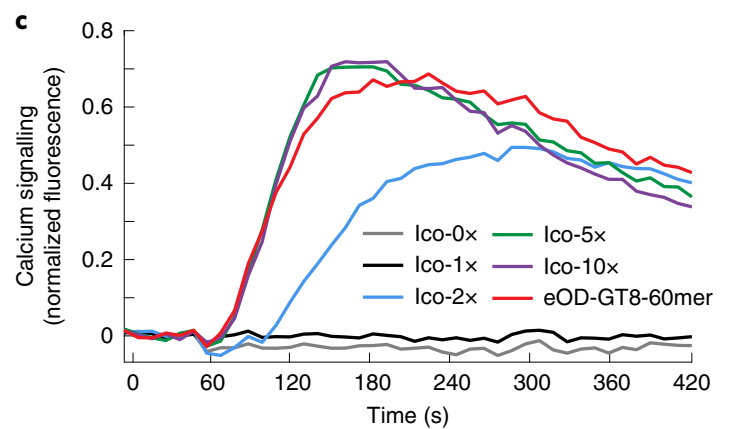

d
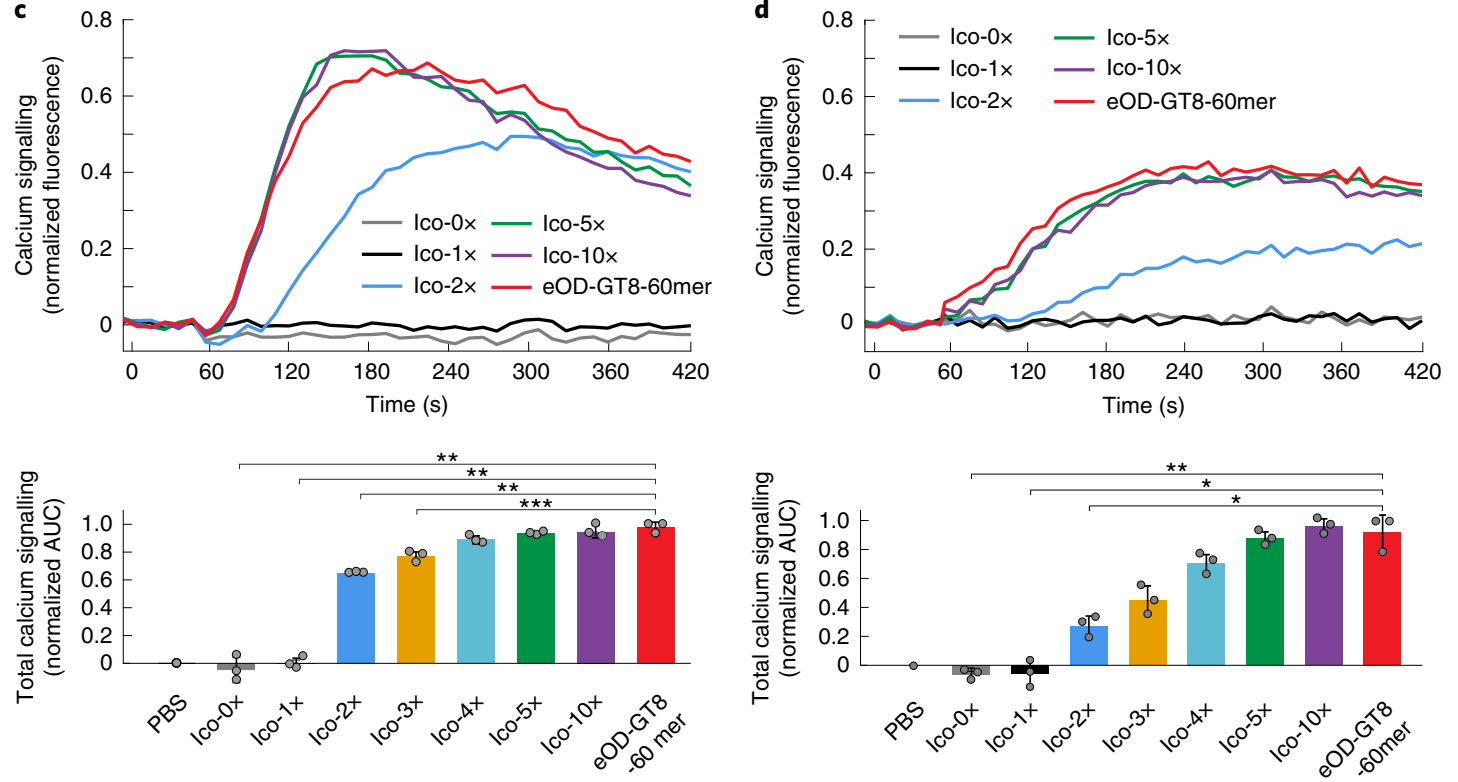

Fig. 2 | Increasing antigen valency improves B-cell responses to NP antigens up to a threshold. a, Folding of the two types of DNA-NPs (six-helix bundle and DNA icosahedron) that were designed and used in this study for 1D and 3D presentations of antigens. The TEM images show high folding yield and monodisperse DNA-NPs. Scale bars, $40 \mathrm{~nm}$. b. Overview of the antigen conjugation protocol to attach eOD-GT8 antigens to the DNA-NPs using PNA single strands complementary to sSDNA overhangs on the DNA-NPs and characterization with electrophoresis. Shown are representative gel electrophoresis samples and fluorescence quantification of four different icosahedral DNA-NPs conjugated with eOD-GT8:PNA-AF647 (Ico-1X; Ico-5X; Ico-30X; Ico-60X); (M, molecular weight marker; Sc, scaffold; bp, base pair). Error bars represent s.d. of the mean ( $n=3$ biological replicates per group for fluorescence quantification). c,d, DNA-NPs modified with eOD-GT8 activate lgM-BCR at both $5 \mathrm{nM}$ (c) and 0.5nM (d) eOD-GT8. Fluo-4 calcium probe fluorescence is shown in the top row (representative individual calcium traces) and average area-under-the-curve (AUC) measurements for calcium signalling normalized to the maximum response of all samples in a repeat are shown in the bottom row. In $\mathbf{c}$ and $\mathbf{d}$, error bars represent s.d. of the mean ( $n=3$ biological replicates per group). $P$ values are from a two-way analysis of variance (ANOVA), paired Student's $t$-test $\left({ }^{\star} P<0.05,{ }^{\star \star} P<0.01\right.$, ${ }^{\star \star \star} P<0.001$; all $P$ values are provided in Supplementary Table 5). The electron microscopy images in a are from three technical replicates (10 images per replicate) with similar results. The gels in $\mathbf{b}$ were repeated three times (biological replicates) with similar results.

spacing tested of $\sim 7 \mathrm{~nm}( \pm 3 \mathrm{~nm}$ linker size $)$ and, surprisingly, subsequently increased monotonically with increasing antigen spacing at fixed total antigen concentration (Fig. 3a). Calcium responses appeared to plateau at an antigen spacing of $\sim 25-30 \mathrm{~nm}$, yet remained elevated at a spacing of $\sim 80 \mathrm{~nm}$, despite the fact that this latter spacing would preclude binding of the rod-like antigen dimer to two closely spaced IgM-BCRs or to a single IgM-BCR, because this distance is well beyond the spatial tolerance anticipated for a single IgM-BCR ${ }^{31}$. Although in this study we did not test dimer distances larger than $80 \mathrm{~nm}$, we confirmed that enhanced triggering by widely spaced dimers was not due to differences in overall IgM-BCR engagement with the nanostructures, because flow cytometry analysis of fluorescent $6 \mathrm{HB}$ association with Ramos cells showed essentially identical binding of 7-nm-spaced eOD dimers and 28-nm-spaced dimers to B cells after a 30-min incubation with the constructs in comparison with the increased level of binding observed for a higher number of antigens presented by the construct Ico-30× (Fig. 3b and Supplementary Fig. 17).

To test the role of fixed inter-antigen spacing imparted by DNANP scaffold rigidity on IgM-BCR triggering by dimeric antigens, we compared calcium signalling induced by rigid DNA origami rods to eOD-GT8 dimers presented using flexible ssDNA or PEG linkers of variable contour lengths (Supplementary Table 1). These flexible polymer constructs presenting eOD-GT8 dimers elicited substantially reduced B-cell signalling compared with their rigid DNANP counterparts, indicating the importance of both inter-antigen 

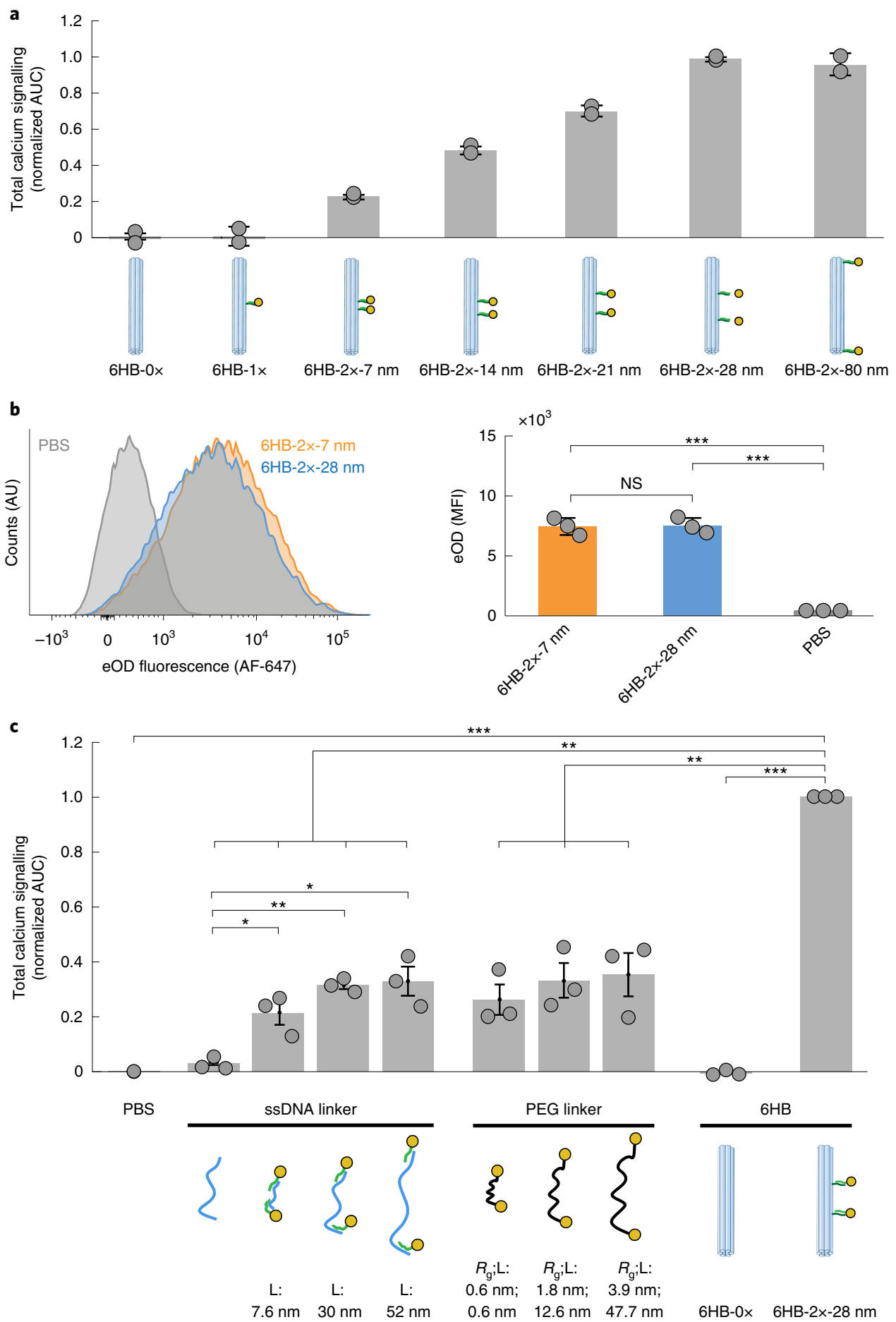

Fig. 3 | IgM-BCR response increases and then plateaus with increasing inter-antigen distance on a rigid scaffold. a, AUC total calcium signalling in gIVRC01 B cells stimulated with DNA-NP eOD-GT8 dimers with inter-antigen distances between $7 \mathrm{~nm}$ and $80 \mathrm{~nm}$ at an antigen concentration of $5 \mathrm{nM}$ ( $n=2$ biological replicates per group). Fluo-4 AUC is normalized as in Fig. 2, where error bars represent s.d. of the mean. b, Representative flow cytometry plots of $6 \mathrm{H}-2 \times-7 \mathrm{~nm}$ and $6 \mathrm{HB}-2 \times-28 \mathrm{~nm}$ binding to antigen-specific $\mathrm{B}$ cells (left) after 30 -min incubation at $4^{\circ} \mathrm{C}$ at a fixed antigen concentration of $5 \mathrm{nM}$. Right: quantitation of data from flow cytometry (MFI, mean fluorescence intensity; $n=3$ distinct biological replicates per group), where error bars represent s.d. of the mean and $P$ values are from a one-way ANOVA, followed by Tukey post hoc comparison test ( ${ }^{\star \star \star} P<0.001$; NS, not significant, $P=0.9999$; all $P$ values are provided in Supplementary Table 6). c, Total calcium release (Fluo-4 fluorescence) integrated over 7 min following antigen addition from cells stimulated with eOD-GT8 dimers attached to the flexible polymeric scaffolds (ssDNA or PEG), compared with rigid 6HB DNA-NP eOD-GT8 dimer structures at a fixed antigen concentration of $5 \mathrm{nM}$ ( $n=3$ distinct biological replicates per group). Fluo- 4 AUC is normalized as in Fig. 2, where error bars represent s.d. of the mean and $P$ values are from a two-way ANOVA, paired Student's $t$-test $\left({ }^{\star} P<0.05,{ }^{\star \star} P<0.01\right.$, ${ }^{\star \star \star} P<0.001$; all $P$ values are provided in Supplementary Table 7). L, contour length; $R_{g^{\prime}}$ radius of gyration. 

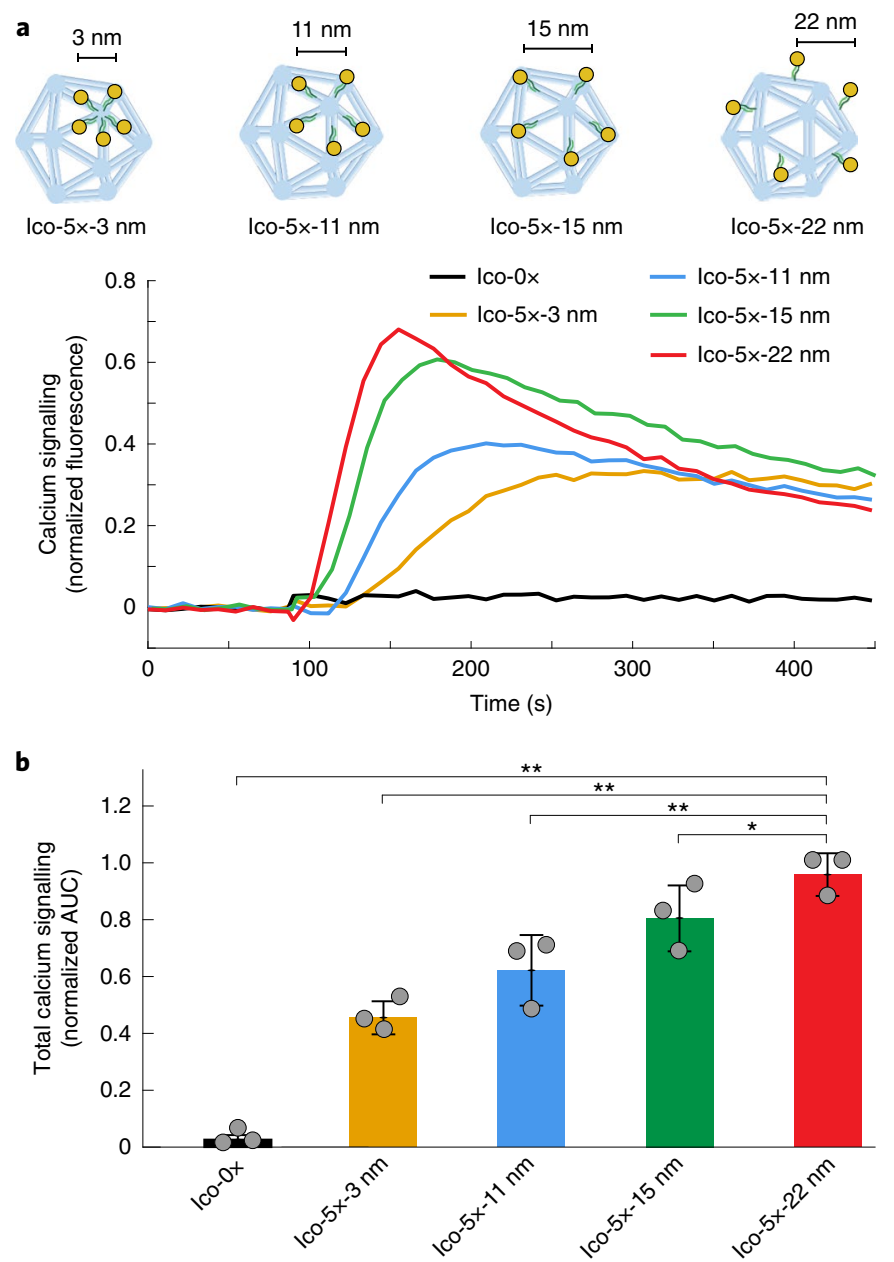

Fig. 4 | Clustering of antigens on one face of an icosahedral DNA-NP. a, Plot of Fluo-4 calcium probe fluorescence versus time following addition of $5 \mathrm{nM}$ eOD-GT8 antigen to gIVRC01 B cells. Icosahedral (Ico) structures with varying inter-antigen distances are shown at the top. Representative individual calcium traces are shown ( $n=3$ biological replicates with similar results). b. Total calcium signalling integrated over 6 min following antigen addition from cells stimulated with different Ico- $5 \times$ structures presenting antigen at a total concentration of $5 \mathrm{nM}$ eOD-GT8 $(n=3$ biological replicates per group). Fluo-4 AUC is normalized as in Fig. 2, where error bars represent s.d. of the mean and $P$ values are from a two-way ANOVA, paired Student's $t$-test $\left({ }^{\star} P<0.05,{ }^{\star}{ }^{\star} P<0.01\right.$; all $P$ values are provided in Supplementary Table 8).

distance and also the rigidity of the structural scaffold used to present the antigens for inducing a robust B-cell response (Fig. 3c).

To further investigate the relative roles of inter-antigen spacing and the dimensionality of presentation on B-cell response, we programmed different clusters of five eOD-GT8 antigens on one face of the icosahedral DNA-NP using distinct inter-antigen distances of $\sim 3$ to $22 \mathrm{~nm}$ (or a maximum inter-antigen spacing of $36 \mathrm{~nm}$ ), and we also varied the DNA origami NP scaffold geometry itself (Fig. 4a,b and Supplementary Figs. 18-20). We found that increasing inter-antigen distances on the DNA icosahedron also led to a monotonic increase in cellular response, similar to that observed with the dimer presented on a rigid-rod origami (Fig. 3). For inter-antigen distances greater than $\sim 22 \mathrm{~nm}$, cellular responses also appeared to plateau (Supplementary Fig. 18), although considerably larger origami objects would be needed to rigorously establish cellular behaviour beyond this observed upper limit. Using the rigid rod to alternatively display five antigens in a linear manner with similar minimum inter-antigen distances showed that decreasing inter-antigen distance also yielded a decreasing cellular response, consistent with our observations with the 6HB dimer DNANP (Supplementary Fig. 18). However, for the smaller minimal inter-antigen distances of 7 and $11 \mathrm{~nm}$, the linear antigen display yielded a relatively higher response than the quasi-planar organization of antigens (Supplementary Fig. 18), probably because of its larger mean or maximal inter-antigen spacings (for example, the $6 \mathrm{HB}-5 \times-11 \mathrm{~nm}$ mean and maximal spacings are $22 \mathrm{~nm}$ and $44 \mathrm{~nm}$; Supplementary Fig. 19).

To test the potential role of 3D DNA-NP geometry on IgM-BCR triggering, we also designed a disk-like pentagonal bipyramid to compare cellular activation with the icosahedron. At an antigen copy number of 10 , we observed similarly robust B-cell activation, comparable with the icosahedron and the eOD-GT8-60mer reference particle (Supplementary Fig. 20). To test the generality of our DNA-NP platform for presentation of other antigens and to evaluate whether similar NPs could activate naive primary B cells, we hybridized the pentagonal bipyramid with short linear peptide antigens, which are recognized by murine 3-83 IgM-BCR-transgenic B cells. ${ }^{32}$ Forty-five copies of medium-affinity (p31, $\left.K_{\mathrm{D}}=15.3 \mathrm{nM}\right)$ or low-affinity ( $\left.55, K_{\mathrm{D}}=1 \mu \mathrm{M}\right)$ peptide antigens were conjugated, and IgM-BCR triggering was compared with presentation by liposomes, a common synthetic vaccine platform. Although activation levels were comparable among all three constructs, IgM-BCR triggering appeared to be slightly delayed in the case of the liposomes, despite the considerably higher total copy number of immunogens, the similar mean inter-antigen spacing $(\sim 7$ versus $11 \mathrm{~nm}$ for the DNA-NP and Lipo, respectively) and the larger overall particle size (Supplementary Fig. 21). This observation further supports the importance of scaffold rigidity and fixed inter-antigen spacing for efficient B-cell triggering, even for low-affinity antigens.

\section{Imaging of early events in DNA-NP binding to B cells}

To probe the mechanism of B-cell activation, we examined three distinct DNA-NP constructs using fluorescence imaging: two $6 \mathrm{HB}$ dimers with inter-antigen spacings of 7 and $28 \mathrm{~nm}$ that respectively triggered low and high $\mathrm{B}$-cell activation, and one DNA icosahedral NP presenting 30 copies of eOD-GT8 that triggered robust B-cell activation. Fluorescently labelled eOD-GT8-presenting DNA-NPs were added to Ramos cells at identical total eOD-GT8 concentrations and spatially correlated with fluorescently labelled IgM-BCR on the cell surface before internalization (Fig. 5a and Supplementary Figs. 22-24). As anticipated, DNA-NP binding was strongly correlated with VRC01 IgM expression, whereas cells lacking IgM expression failed to bind the eOD-GT8-bearing particles (Fig. 5b and Supplementary Figs. 22-24). For all DNA-NP constructs examined, antibody staining of phosphorylated Syk kinase (pSyk) revealed a sharp increase in pSyk phosphorylation after only $1 \mathrm{~min}$ of DNA-NP or eOD-GT8-60mer addition. Moreover, dimers of eOD-GT8 separated by short distances of only $7 \mathrm{~nm}(6 \mathrm{HB}-2 \times-7 \mathrm{~nm})$ resulted in significantly lower pSyk accumulation compared with dimers separated by larger distances of $28 \mathrm{~nm}(6 \mathrm{HB}-2 \times-28 \mathrm{~nm})$ or icosahedral NPs bearing 30 copies of eOD-GT8 (Ico-30X) (Fig. 5c). Characterization of the internalization of eOD using phalloidin to stain filamentous actin in the actin-rich cellular cortex indicated greater internalization of the icosahedral 30-mer NP compared with its $6 \mathrm{HB}-2 \times-7 \mathrm{~nm}$ and $6 \mathrm{HB}-$ $2 \times-28 \mathrm{~nm}$ counterparts (Fig. $5 \mathrm{~d}$ ). Taken together, these results corroborated both the levels of cellular activation observed for these three DNA-NP constructs, and that the previously observed calcium responses were acting through the IgM-BCR-mediated activation pathway that includes pSyk phosphorylation and downstream NP internalization. 

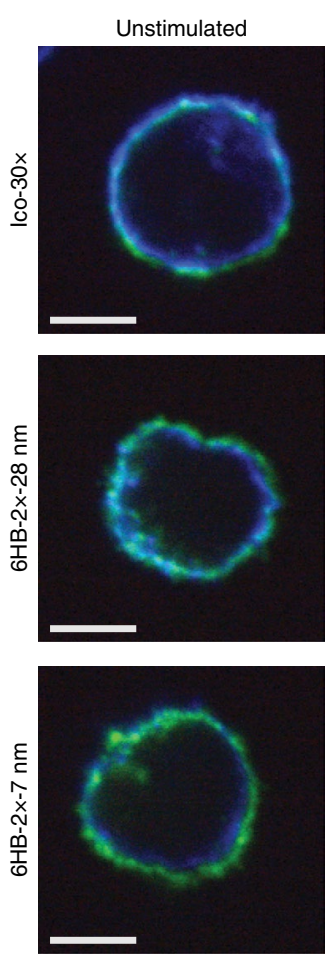
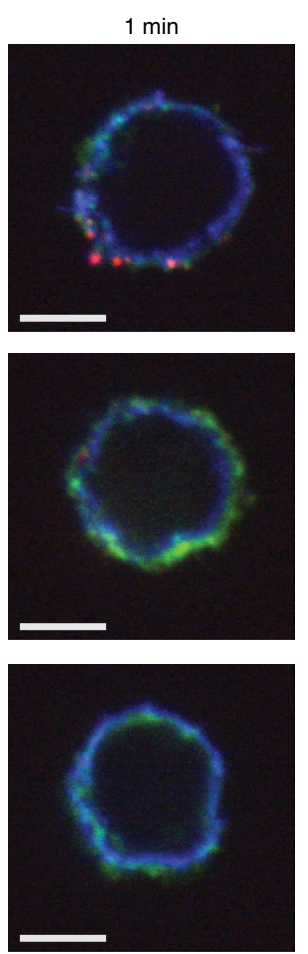
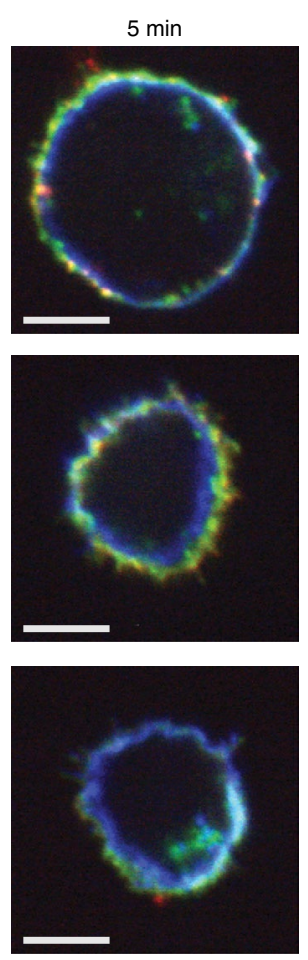

$30 \mathrm{~min}$
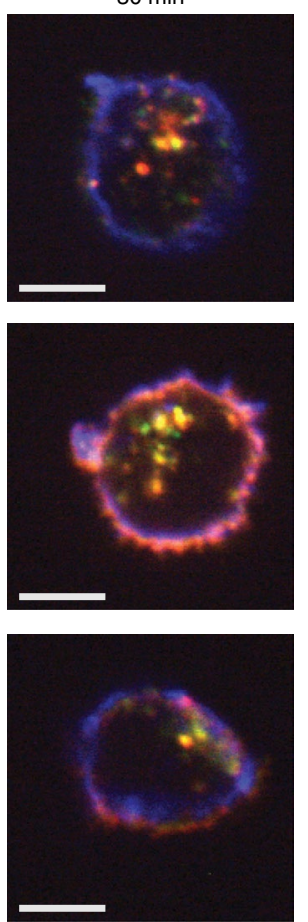
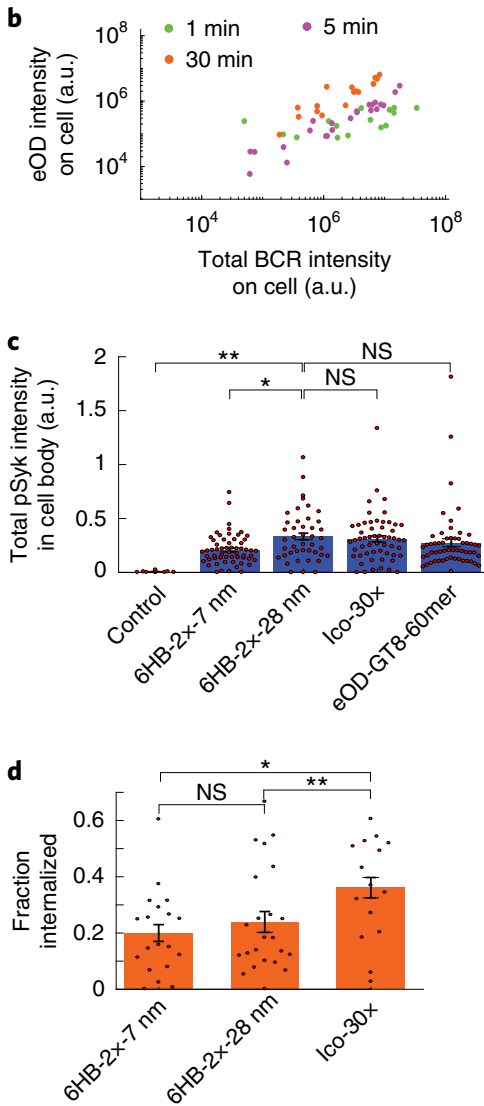

Fig. 5 | Confocal microscopy imaging of DNA-NPs on Ramos B cells. a, Time series imaging of Ico-30×, $6 \mathrm{HB}-2 \times-28 \mathrm{~nm}$ and $6 \mathrm{HB}-2 \times-7 \mathrm{~nm}$ shows surface binding and internalization into Ramos B cells. Here, eOD-GT8 was fluorescently labelled with AF647, VRC01 IgM-BCR was labelled with a FAb fragment conjugated to Janelia Fluor 549 before antigen addition, and actin was labelled with phalloidin Alexa Fluor 405 after cell fixation. Scale bars, $5 \mu$ m. The experiment was performed twice ( $n=2$ biological replicates) with similar results. $\mathbf{b}$, The total intensity of eOD-GT8 is highly correlated with the intensity of IgM-BCR, confirming specific binding of NPs to the IgM-BCR and co-internalization. The numbers of cells analysed are 15 (1 min), 23 (5 min) and 16 (30 min). Cells are from the same culture. c, Ramos cells were labelled with an anti-phospho-Syk antibody after fixation and the total pSyk intensity per cell was determined. The numbers of cells analysed are 11 (control), 58 (6HB-2x-7 nm), 43 (6HB-2x-28 nm), 56 (Ico-30x) and 56 (eOD-GT8-60 mer). Cells are from the same culture. $\mathbf{d}$, The internalized fraction of eOD was estimated by segmenting the cell surface using a phalloidin stain, as detailed in the Methods. The total internal eOD fluorescence was divided by total cellular eOD fluorescence on a cell-by-cell basis. The numbers of cells analysed are 19 (6HB-2x-7 nm), 23 (6HB-2x-28 nm) and 15 (Ico-30x). Cells are from the same culture. In c and $\mathbf{d}$, error bars denote the s.e.m. fluorescence between cells, with significance determined by a two-sided Student's $t$-test $\left({ }^{\star} P=0.0314,{ }^{\star \star} P=0.0016\right.$; NS, Not statistically significant; $P=0.3038$ for $6 \mathrm{HB}-2 \times-28$ nm/Ico$30 \times ; P=0.4153$ for $6 \mathrm{HB}-2 \times-28 \mathrm{~nm} / \mathrm{eOD}-\mathrm{GT} 8-60$ mer). In d error bars denote the standard error of the mean fluorescence between cells with significance determined by Student's t-test ( ${ }^{\star} P=0.0142,{ }^{\star \star} P=0.0074 ;$ NS, not significant, $P=0.55$ ). All $P$ values are provided in Supplementary Table 9 .

\section{Modelling of early BCR signalling after B-cell triggering by DNA-NPs}

Consistent with previous studies and models of B-cell triggering, we observed that the nanometre-scale distances between the immunogens and their valency ${ }^{2,3,6}$ are both crucial determinants of IgM-BCR activation and cellular response $\mathrm{e}^{27-30,33}$. However, our results also point clearly to the importance of scaffold rigidity to maintain inter-antigen separations for optimal IgM-BCR activation. Interestingly, we observed a monotonic increase in cellular activation in both linear and quasi-planar spatial antigen presentations up to a spacing of $\sim 25-30 \mathrm{~nm}$ (Supplementary Fig. 18). In addition, our results showed sustained, efficient signalling induction for an $80-\mathrm{nm}$ dimer (Fig. 3a), suggesting that antigen engagement of non-local, distal B-cell receptors can also drive the initiation of signalling in response to antigen binding. Although these non-local interactions may stem from a variety of sources that would require sophisticated single-molecule imaging and molecular perturbation analyses to dissect, two discrete possibilities include signalling cooperativity between the antigen receptor and slowly evolving structural elements within the B-cell membrane $\mathrm{e}^{34}$ and the actin cortex ${ }^{35}$, which could facilitate communication of receptor binding over such large distances ${ }^{28}$, or spatially mediated positive feedback between signalling components downstream of IgM-BCR, as explored in previous work ${ }^{36}$.

To explore the latter hypothesis theoretically, we applied an in silico coarse-grained reaction-diffusion model to our system that was previously used to describe BCR signalling (Supplementary Text 1 and Supplementary Figs. 25 and 26) ${ }^{36}$. The model describes early-time membrane-proximal IgM-BCR signalling initiated by IgM-BCR antigen binding of glVRC01 IgM to eOD-GT8 antigens, followed by immunoreceptor tyrosine-based activation motif (ITAM) phosphorylation mediated by reaction-diffusion of Lyn and Syk, where antigen unbinding events are neglected due to the slow off-rate of this antigen/receptor pair $\left(k_{\text {off }}<5.05 \times 10^{-5} \mathrm{~s}^{-1}\right)^{20}$. This model is agnostic towards the competing mechanisms previously proposed for BCR triggering ${ }^{37}$, namely disintegration of auto-inhibited BCR multimers upon antigen binding into smaller active BCR clusters $^{27}$, formation of BCR clusters due to 
conformational changes in antigen-binding $\mathrm{BCRs}^{30,38}$ or actin-mediated restriction of BCR mobility limiting ITAM phosphorylation $^{33}$. In the model, monomeric IgM-BCRs engage with each antigen on the origami, where IgM-BCR clusters are formed at the nanoscale $(\sim 30 \mathrm{~nm})$ upon binding (Supplementary Text 1), as justified by several experiments ${ }^{29,37}$ including super-resolution microscopy ${ }^{29,39}$, as well as our own data (Supplementary Figs. 23 and 24). Although our model does not propose any specific mechanism for this clustering, it explores the consequence of such spatial clustering on B-cell activation through IgM-BCR. Our results show (Supplementary Text 1 and Supplementary Fig. 25) that antigens separated by less than $30 \mathrm{~nm}$ limit the total amount of IgM-BCRs recruited to each antigen due to steric exclusion. Thus, as inter-antigen separation increases, IgM-BCR signalling increases until the IgM-BCR clusters are no longer overlapping, resulting in a signalling plateau (Supplementary Fig. 26). A similar plateau was observed theoretically for lower-affinity antigen, albeit at a lower level of activation, as observed experimentally for the lower-affinity antigen variant eOD-GT5 (Supplementary Fig. 16, Supplementary Text 1 and Supplementary Figs. 25 and 26). Furthermore, while a similar monotonic increase in signalling was observed in silico for increasing minimum inter-antigen spacing in a planar, $2 \mathrm{D}$ pentameric spatial organization, saturation in cellular activation was observed experimentally in the linear pentameric construct at smaller minimum inter-antigen spacings than in the model. Thus, although this model offers a potential mechanism by which monotonic increase in B-cell activation arises as inter-antigen spacing increases, additional factors must limit cellular activation once any inter-antigen distances of $\sim 25-30 \mathrm{~nm}$ are attained.

\section{Conclusions}

In summary, using DNA origami as a platform for controlling the spatial presentation of eOD-GT8 antigens, we identify here several design criteria maximizing early B-cell triggering, including a valency of five or more antigens, nearest-neighbour spacings of $\sim 25-30 \mathrm{~nm}$ or greater and use of a rigid scaffold for antigen display. In the future, additional modelling studies may be combined with advanced super-resolution and single-molecule imaging to explore alternative, competing hypotheses such as the presence of actin 'corrals' that may also contribute to explaining these experimental observations. In addition, the relative roles of monomeric versus dimeric binding to individual IgM-BCRs based on the spatial tolerance of IgM, which is not captured in our model, would also be interesting to explore with single-particle-based, stochastic models ${ }^{31}$. Finally, affinity is also an important determination of $\mathrm{BCR}$ responses ${ }^{32,40}$. We reiterate that the affinity between germline VRCO1 and eOD-GT8 is in the subnanomolar range, placing this system in the regime of mature IgM-BCR antigen affinities, rather than naive affinities. In the case of lower-affinity interactions, as explored for the eOD-GT5 and model peptide antigens, higher multivalency might play a more prominent role in B-cell activation. Indeed, it has been suggested that the low number of Env viral spikes displayed on the viral surface of HIV may help it to evade detection by the immune system ${ }^{41}$. Notwithstanding this, for the purpose of the rational design of molecular vaccines for robust triggering of B-cell responses, here DNA origami has offered crucial insight into the spatial relationships between immunogens, which may be generalized to other viral pathogens such as SARS-CoV-2 and Zika, and offered important foundations for the rational design of protein-based and other vaccine platforms ${ }^{42}$.

\section{Online content}

Any methods, additional references, Nature Research reporting summaries, source data, extended data, supplementary information, acknowledgements, peer review information; details of author contributions and competing interests; and statements of data and code availability are available at https://doi.org/10.1038/ s41565-020-0719-0.

Received: 12 June 2019; Accepted: 27 May 2020;

Published online: 29 June 2020

\section{References}

1. Bachmann, M. F. \& Jennings, G. T. Vaccine delivery: a matter of size, geometry, kinetics and molecular patterns. Nat. Rev. Immunol. 10, 787-796 (2010).

2. Puffer, E. B., Pontrello, J. K., Hollenbeck, J. J., Kink, J. A. \& Kiessling, L. L. Activating B cell signaling with defined multivalent ligands. ACS Chem. Biol. 2, 252-262 (2007).

3. Minguet, S., Dopfer, E.-P. \& Schamel, W. W. A. Low-valency, but not monovalent, antigens trigger the B-cell antigen receptor (BCR). Int. Immunol. 22, 205-212 (2010).

4. Batista, F. D., Iber, D. \& Neuberger, M. S. B cells acquire antigen from target cells after synapse formation. Nature 411, 489-494 (2001).

5. Dintzis, H. M., Dintzis, R. Z. \& Vogelstein, B. Molecular determinants of immunogenicity: the immunon model of immune response. Proc. Natl Acad. Sci. USA 73, 3671-3675 (1976).

6. Brouwer, P. J. M. et al. Enhancing and shaping the immunogenicity of native-like HIV-1 envelope trimers with a two-component protein nanoparticle. Nat. Commun. 10, 4272 (2019).

7. Steichen, J. M. et al. HIV vaccine design to target germline precursors of glycan-dependent broadly neutralizing antibodies. Immunity 45, 483-496 (2016)

8. Abbott, R. K. et al. Precursor frequency and affinity determine B cell competitive fitness in germinal centers, tested with germline-targeting HIV vaccine immunogens. Immunity 48, 133-146.e6 (2018).

9. Stamatatos, L., Pancera, M. \& McGuire, A. T. Germline-targeting immunogens. Immunol. Rev. 275, 203-216 (2017).

10. Steichen, J. M. et al. A generalized HIV vaccine design strategy for priming of broadly neutralizing antibody responses. Science 366, eaax4380 (2019).

11. Saunders, K. O. et al. Targeted selection of HIV-specific antibody mutations by engineering B cell maturation. Science 366, eaay7199 (2019).

12. Jardine, J. G. et al. Priming a broadly neutralizing antibody response to HIV-1 using a germline-targeting immunogen. Science 349, 156-161 (2015).

13. Sok, D. et al. Priming HIV-1 broadly neutralizing antibody precursors in human Ig loci transgenic mice. Science 353, 1557-1560 (2016).

14. Kim, Y.-M. et al. Monovalent ligation of the B cell receptor induces receptor activation but fails to promote antigen presentation. Proc. Natl Acad. Sci. USA 103, 3327-3332 (2006).

15. Bennett, N. R., Zwick, D. B., Courtney, A. H. \& Kiessling, L. L. Multivalent antigens for promoting B and T cell activation. ACS Chem. Biol. 10, 1817-1824 (2015).

16. Bachmann, M. F. et al. The influence of antigen organization on B cell responsiveness. Science 262, 1448-1451 (1993).

17. Rothemund, P. W. K. Folding DNA to create nanoscale shapes and patterns. Nature 440, 297-302 (2006).

18. Veneziano, R. et al. Designer nanoscale DNA assemblies programmed from the top down. Science 352, 1534-1534 (2016).

19. Wamhoff, E.-C. et al. Programming structured DNA assemblies to probe biophysical processes. Annu. Rev. Biophys. 48, 395-419 (2019).

20. Jardine, J. G. et al. HIV-1 broadly neutralizing antibody precursor B cells revealed by germline-targeting immunogen. Science 351, 1458-1463 (2016).

21. Jardine, J. et al. Rational HIV immunogen design to target specific germline B cell receptors. Science 340, 711-716 (2013).

22. Havenar-Daughton, C. et al. The human naive B cell repertoire contains distinct subclasses for a germline-targeting HIV-1 vaccine immunogen. Sci. Transl. Med. 10, eaat0381 (2018).

23. Schwarz, F. P., Robinson, S. \& Butler, J. M. Thermodynamic comparison of PNA/DNA and DNA/DNA hybridization reactions at ambient temperature. Nucleic Acids Res. 27, 4792-4800 (1999).

24. Scharenberg, A. M., Humphries, L. A. \& Rawlings, D. J. Calcium signalling and cell-fate choice in B cells. Nat. Rev. Immunol. 7, 778-789 (2007).

25. Sil, D., Lee, J. B., Luo, D., Holowka, D. \& Baird, B. Trivalent ligands with rigid DNA spacers reveal structural requirements for IgE receptor signaling in RBL mast cells. ACS Chem. Biol. 2, 674-684 (2007).

26. Cochran, J. R., Cameron, T. O., Stone, J. D., Lubetsky, J. B. \& Stern, L. J. Receptor proximity, not intermolecular orientation, is critical for triggering T-cell activation. J. Biol. Chem. 276, 28068-28074 (2001).

27. Yang, J. \& Reth, M. Oligomeric organization of the B-cell antigen receptor on resting cells. Nature 467, 465-469 (2010).

28. Liu, W., Wang, H. \& Xu, C. Antigen receptor nanoclusters: small units with big functions. Trends Immunol. 37, 680-689 (2016).

29. Gold, M. R. \& Reth, M. G. Antigen receptor function in the context of the nanoscale organization of the B cell membrane. Annu. Rev. Immunol. 37, 97-123 (2019) 
30. Tolar, P. \& Pierce, S. K. in Immunological Synapse (eds Saito, T. \& Batista, F. D.) Vol. 340, 155-169 (Springer, 2010).

31. Shaw, A. et al. Binding to nanopatterned antigens is dominated by the spatial tolerance of antibodies. Nat. Nanotechnol. 14, 184-190 (2019).

32. Kouskoff, V. et al. Antigens varying in affinity for the B cell receptor induce differential B lymphocyte responses. J. Exp. Med. 188, 1453-1464 (1998).

33. Harwood, N. E. \& Batista, F. D. Early events in B cell activation. Annu. Rev. Immunol. 28, 185-210 (2010).

34. Stone, M. B., Shelby, S. A., Núñez, M. F., Wisser, K. \& Veatch, S. L. Protein sorting by lipid phase-like domains supports emergent signaling function in B lymphocyte plasma membranes. eLife 6, e19891 (2017).

35. Tolar, P. Cytoskeletal control of B cell responses to antigens. Nat. Rev. Immunol. 17, 621-634 (2017).

36. Mukherjee, S. et al. Monovalent and multivalent ligation of the B cell receptor exhibit differential dependence upon Syk and Src family kinases. Sci. Signal. 6, ral (2013).

37. Kläsener, K., Maity, P. C., Hobeika, E., Yang, J. \& Reth, M. B cell activation involves nanoscale receptor reorganizations and inside-out signaling by Syk. eLife 3, e02069 (2014).
38. Tolar, P., Hanna, J., Krueger, P. D. \& Pierce, S. K. The constant region of the membrane immunoglobulin mediates B cell-receptor clustering and signaling in response to membrane antigens. Immunity 30, 44-55 (2009).

39. Lee, J., Sengupta, P., Brzostowski, J., Lippincott-Schwartz, J. \& Pierce, S. K. The nanoscale spatial organization of B-cell receptors on immunoglobulin M- and G-expressing human B-cells. Mol. Biol. Cell 28, 511-523 (2017).

40. Liu, W., Meckel, T., Tolar, P., Sohn, H. W. \& Pierce, S. K. Antigen affinity discrimination is an intrinsic function of the B cell receptor. J. Exp. Med. 207, 1095-1111 (2010).

41. Zhu, P. et al. Distribution and three-dimensional structure of AIDS virus envelope spikes. Nature 441, 847-852 (2006).

42. Marcandalli, J. et al. Induction of potent neutralizing antibody responses by a designed protein nanoparticle vaccine for respiratory syncytial virus. Cell 176, 1420-1431.e17 (2019).

Publisher's note Springer Nature remains neutral with regard to jurisdictional claims in published maps and institutional affiliations.

๑) The Author(s), under exclusive licence to Springer Nature Limited 2020 


\section{Methods}

Chemicals and kits. Magnesium chloride, Tris acetate EDTA (TAE) buffer, Tris-base, sodium chloride, phosphate buffered saline (PBS), ethidium bromide (EtBr) solution $\left(10 \mathrm{mg} \mathrm{ml}^{-1}\right)$, Pluronic F-127 (cat. no. 540025-50 ML) and Amicon ultra 0.5 centrifugal filter (cat. no. UFC5003) were provided by Sigma-Aldrich. Nuclease-free water was purchased from Integrated DNA Technologies (IDT). The DNTPs mix (cat. no. N0447S) and DNA ladder (Quick-Load Purple 2-Log DNA ladder 0.1-10 kb, cat. no. N0550S) were provided by New England Biolabs (NEB). The polymerase enzyme (Accustart Taq DNA polymerase HiFi, cat. no. 95085-05K) was provided by Quanta Biosciences. Low-melt agarose was purchased from IBI Scientific (cat. no. IB70058) and the agarose from Seakem. G-capsule for electroelution (cat. no. 786-001) was purchased from G-Biosciences and Freeze'N Squeeze DNA gel extraction columns from Bio-Rad (cat. no. 732-6165). The Zymoclean Gel DNA recovery kit (cat. no. D4008) was purchased from Zymo Research. The SybrSafe DNA staining reagent was provided by ThermoFisher Scientific. PEG3500 (cat. no. A4010-1/MAL-PEG3500-MAL) and PEG2000 (cat. no. A4010-1/MAL-PEG2000-MAL) bismaleimide were purchased from JenKem Technology.

Oligonucleotides and DNA templates. All oligonucleotides used for asymmetric PCR (aPCR) amplification of the template and for folding the various scaffolded DNA origami NPs were purchased from IDT. The circular plasmid DNA scaffold M13mp18 used for amplification of the short scaffolds with aPCR (supplementary sequences are provided in Supplementary Tables 10-17) was acquired from NEB (cat. no. N4040S).

Antigens and cell lines. The eOD antigen with a $6 \mathrm{xHis}$ tag and $\mathrm{N}$-terminal cysteine was prepared as previously described ${ }^{20}$. Plasmids were transiently transfected into Expi293 cells (ThermoFisher Scientific, not authenticated). After five days, cell culture supernatants were collected and protein was purified in an ÄKTA pure chromatography system using a HiTrap HP Ni sepharose affinity column, followed by size exclusion chromatography using a Superdex 75 Increase 10/300 GL column (GE Healthcare Life Sciences). Endotoxin levels in purified protein were measured using an Endosafe Nexgen-PTS system (Charles River) and assured to be $<5$ endotoxin units (EU) per $\mathrm{mg}$ protein. PNA-conjugated peptide antigens p31 (HDWRSGFGGFQHLCC-O-Linker-cagtccagt-K(AF-647)) and p5 (SGSVTYLPTPEWALQSGS-O-Linker-cagtccagt-K(AF-647)) were purchased from PNA Bio. Ramos B cells stably expressing VRC01 germline IgM B-cell receptors were provided by D. Lingwood (Ragon Institute of MGH, MIT and Harvard) ${ }^{43,44}$. As described previously, VRC01 germline cells were generated by stable lentiviral transduction of surface IgM-negative Ramos B cells and IgM-BCR-expressing cells were sorted by flow cytometry. Antigen-specific receptor expression levels after transduction were characterized previously and found to be $\sim 12,000$ per $\mathrm{cell}^{14}$. Functional expression of germline VRC01 was confirmed by flow cytometry analysis of labelled eOD probes binding to the VRC01 Ramos cells. Both Expi293 and germline VRC01-expressing Ramos B cells tested negative for mycoplasma.

ssDNA scaffold synthesis. The ssDNA scaffolds used to fold the DNA $6 \mathrm{HB}$ and the DNA icosahedron NPs were produced using a previously described procedure with asymmetric $\mathrm{PCR}^{18,45}$. Briefly, two specific primer sets were used to amplify the ssDNA fragments (Supplementary Table 3) using Quanta Accustart HiFi DNA polymerase. The aPCR mix was prepared to a final volume of $50 \mu$ with the specific polymerase buffer complemented with $2 \mathrm{mM}$ magnesium chloride, $200 \mu \mathrm{M}$ dNTPs, $1 \mu \mathrm{M}$ forward primer, $20 \mathrm{nM}$ reverse primer, $25 \mathrm{ng}$ M13mp18 template and 1 unit of Quanta Accustart HiFi polymerase. The amplification protocol was as follows: $94^{\circ} \mathrm{C}$ for $1 \mathrm{~min}$ for initial denaturation followed by 35 cycles of $94^{\circ} \mathrm{C}$ held for $20 \mathrm{~s} ; 56^{\circ} \mathrm{C}$ held for $30 \mathrm{~s} ; 68^{\circ} \mathrm{C}$ held for $1 \mathrm{~min}$ per kb for amplification. Following amplification, the aPCR mix was run on a $1 \%$ low-melt agarose gel prestained with EtBr. The resulting ssDNA product was then extracted using a Zymoclean gel DNA recovery kit. The custom circular DNA scaffold phPB84 used for the pentagonal bipyramid DNA-NPs was prepared as previously published ${ }^{46}$. The purified ssDNA concentration was measured using a NanoDrop 2000 (Thermo Scientific).

DNA-NP folding. DNA-NPs (icosahedron, pentagonal bipyramid and 6HB), with or without overhangs, were self-assembled using a one-pot reaction and annealing as described previously ${ }^{17,18}$. Briefly, $20-40 \mathrm{nM}$ of scaffold was mixed with an excess of the staple strand mix (molar ratio of $10 \times$ ) in buffer TAE- $\mathrm{MgCl}_{2}(40 \mathrm{mM}$ Tris, $20 \mathrm{mM}$ acetic acid, $2 \mathrm{mM}$ EDTA, $16 \mathrm{mM} \mathrm{MgCl}_{2}, \mathrm{pH} 8.0$ ) in a final reaction volume of $50 \mu \mathrm{l}$ and annealed with the following program: $95^{\circ} \mathrm{C}$ for $5 \mathrm{~min}, 80-75^{\circ} \mathrm{C}$ at $1{ }^{\circ} \mathrm{C}$ per $5 \mathrm{~min}, 75-30^{\circ} \mathrm{C}$ at $1^{\circ} \mathrm{C}$ per $15 \mathrm{~min}$ and $30-25^{\circ} \mathrm{C}$ at $1^{\circ} \mathrm{C}$ per $10 \mathrm{~min}$. For the pentagonal bipyramid, the reverse-complement oligonucleotide to the overhang sequence was added to the reaction mixture at twofold excess over the total concentration of the overhang sequence. The folded NPs were stored at $4^{\circ} \mathrm{C}$ in the folding buffer with an excess of staple strands before performing conjugation with antigens.

DNA-NP purification. Before using the DNA-NPs for conjugation with antigens and for the B-cell activation assay, the DNA origami objects folded with an excess of staple strands were purified using an Amicon ultra 0.5 centrifugal filter with three washes of folding buffer and an extra wash of $1 \times$ PBS for further modification with antigens. For the pentagonal bipyramid, DNA-NPs with overhangs were purified into TAE- $\mathrm{MgCl}_{2}$ buffer before functionalization with antigen and concentrated to at least fivefold over the target concentration for the functionalization reaction. Centrifugation steps were performed at $1,000 \mathrm{~g}$ for $30-40 \mathrm{~min}$ and the final concentration of NPs was determined using a NanoDrop 2000 system. Purified NPs were subsequently modified with antigens or stored in $1 \times$ PBS (or TAE- $\mathrm{MgCl}_{2}$ buffer) at $4^{\circ} \mathrm{C}$.

PNA strand synthesis. PNA strands were synthesized in house using solid-phase peptide synthesis. Lysine residues were attached at either end of the PNA sequence to improve solubility. Fmoc-PNA monomers (PNA-Bio) were coupled to a low-loading Tentagel-S-RAM resin using 4 equiv. PNA, 3.95 equiv. benzotriazol1-yl-oxytripyrrolidinophosphonium hexafluorophosphate (РyBOP) and 6 equiv. diisopropylethylamine (DIEA). Lysine and glycine residues were reacted in the same way. Following each coupling, the peptide was deprotected in $20 \%$ piperidine in dimethylformamide (DMF). $N$-maleoyl- $\beta$-alanine (Sigma) was coupled to the $\mathrm{N}$ terminus under the same coupling conditions. The peptide was then cleaved from the resin in $95 \%$ trifluoroacetic acid (TFA), $2.5 \% \mathrm{H}_{2} \mathrm{O}$ and $2.5 \%$ triisopropylsilane. The peptide was dissolved in aqueous solution with $0.1 \%$ TFA, filtered and purified by high-performance liquid chromatography using a C-18 Gemini column (Phenomenex) with a mobile phase of acetonitrile containing $0.1 \%$ TFA. The purity of the PNA products was analysed with matrix-assisted laser desorption/ ionization-time of flight mass spectrometry on a Bruker Daltonics microflex. The sequence of the synthesized PNA strand was (maleimide)-GGK-cagtccagt-K$\left(\mathrm{CONH}_{2}\right)$, and the complementary ssDNA was $5^{\prime}$-oligo-TT-ACTGGACTG-3' (predicted melting temperature of $56.7^{\circ} \mathrm{C}$ ). The sequence was designed to have a melting temperature above $55^{\circ} \mathrm{C}$ (predicted with the PNA tool https://www.pnabio. com/support/PNA_Tool.htm, from PNA Bio) and to be orthogonal to the sequence of M13mp18, and was validated using the NCBI BLAST online tool.

Antigen-PNA conjugation. PNA strands were conjugated to eOD by reacting the terminal maleimide onto an $\mathrm{N}$-terminal cysteine of eOD. Before the reaction, eOD was incubated with a tenfold molar excess of tris(2-carboxyethyl)phosphine (TCEP) for $15 \mathrm{~min}$, after which the TCEP was removed using a centrifugal filter. Immediately after removal of TCEP, a twofold molar excess of maleimide-PNA was reacted with cysteine-eOD overnight at $4^{\circ} \mathrm{C}$ in PBS. Unreacted PNA was then removed using an Amicon centrifugal filter $(10 \mathrm{kDa}$ molecular weight cut-off (MWCO)).

Antigen conjugation with AF647 dye. The eOD-PNA conjugate was modified with the fluorescent label AF647-NHS (AF647) using a protocol previously published in ref. ${ }^{47}$. Briefly, eOD-PNA was incubated with 5 molar equiv. of AF647-NHS in $10 \mathrm{mM}$ sodium bicarbonate buffer for $2 \mathrm{~h}$ at room temperature. Unreacted dye was removed using centrifugal filtration (10 kDa MWCO).

Antigen attachment to DNA-NPs. Purified DNA-NPs were mixed with PNA-antigen conjugates at a molar ratio of $5 \times$ antigen per overhang on the DNA-NPs in $1 \times$ PBS buffer. The concentration of DNA-NPs used was in the range of $50-100 \mathrm{nM}$. An annealing temperature ramp was used for ssPNA-ssDNA hybridization, starting at $37^{\circ} \mathrm{C}$ and decreasing to $4^{\circ} \mathrm{C}$ at $1{ }^{\circ} \mathrm{C}$ per $20 \mathrm{~min}$ and kept for at least $4 \mathrm{~h}$ at $4^{\circ} \mathrm{C}$ before use in the B-cell activation assay. Before use in the activation assay, modified DNA-NPs were purified using a centrifugal filter, as described in the DNA-NP purification section, to remove excess free PNA-antigen. Peptide antigens were added from DMF stock solutions and maximal target concentrations of DMF in the functionalization reaction were kept below 5\% (vol/ $\mathrm{vol}$ ). For purification of the functionalized pentagonal bipyramid, centrifugal filters were coated with Pluronic F-127.

Structural characterization. TEM. DNA-NPs were visualized by TEM using grids prepared as described previously, with minor modifications ${ }^{48}$. Briefly, carbon-supported grids with copper mesh (CF200H-CU, Electron Microscopy Sciences) were glow discharged and soaked in $100 \mu \mathrm{M} \mathrm{MgCl}_{2}$ and blotted before depositing DNA-NPs. A 20- $\mu$ l volume of $10 \mathrm{nM}$ DNA-NP solution was applied to a clean parafilm surface and the grid was floated for $2 \mathrm{~min}$. While soaking, $2 \%$ uranyl formate (UF, Electron Microscopy Sciences) was neutralized with $25 \mathrm{mM}$ $\mathrm{NaOH}$ (final concentration), vortexed for $1 \mathrm{~min}$ and filtered via syringe through a $0.1-\mu \mathrm{m}$ filter (EMD Millipore), dropwise, onto the clean parafilm surface. The grid was then removed and quickly dried by edge blotting with Whatman 44 ashless paper. The grid was immediately transferred to the $2 \%$ UF solution and incubated for $30 \mathrm{~s}$. Again, the grid was dried by blotting along the edge with Whatman paper, then left to dry in air for an additional $30 \mathrm{~min}$ before imaging. Imaging was performed on a FEI Tecnai G2 Spirit TWIN set to $120 \mathrm{kV}$ equipped with a Gatan camera. Images were acquired at $\times 6,500$ for wide-field views and $\times 52,000$ for near-field views. Images were collected using 3-s exposures. All raw images were cropped in Adobe Photoshop with subsequent autocontrast applied.

Agarose gel electrophoresis. DNA-NPs folded and conjugated with eOD-GT8-PNA were analysed using agarose gel electrophoresis with $2 \%$ agarose gel prestained 
with EtBr. Samples, non-purified in folding buffer or purified in PBS buffer, were loaded at a concentration of $20-50 \mathrm{nM}$ of DNA origami, run for $2-3 \mathrm{~h}$ at $70 \mathrm{~V}$ at $4{ }^{\circ} \mathrm{C}$ and visualized with a transilluminator. For fluorescence gel analysis with AF647, modified eOD-GT8 images were acquired using a Typhoon FLA 7000 scanner at the SybrSafe excitation wavelength $(473 \mathrm{~nm})$ and the AF647 excitation wavelength $(635 \mathrm{~nm})$. Images were subsequently merged using ImageJ software ${ }^{49}$.

Fluorescence quantification of DNA-NP coverage with antigen. Quantification of the eOD-GT8 conjugation to DNA-NPs was performed using a Fluoromax-4 (Horiba) fluorimeter. eOD-GT8-PNA monomers were modified with AF647 dye using NHS- $\mathrm{NH}_{2}$ chemistry as described above, before conjugation via hybridization to DNA-NPs. eOD-GT8-PNA was incubated with 5 molar equiv. of AF647 for $2 \mathrm{~h}$, and subsequently purified using centrifugal spin filtration (10,000 MWCO). The degree of labelling was two dyes per protein on average. Spectra were acquired with an excitation wavelength of $630 \mathrm{~nm}$ (emission measured at $670 \mathrm{~nm}$ ). A fluorescence calibration curve was first measured using free monomeric eOD-GT8-PNA conjugated with AF647 dye by varying the antigen concentration, and subsequently used as a reference curve to determine the conjugation yield to DNA-NPs.

Tryptophan assay for quantification of DNA-NP coverage with antigen. A tryptophan fluorescence standard curve $(0-2 \mu \mathrm{M})$ was used to determine the percentage of antigen coverage on DNA-NPs. Tryptophan fluorescence was read on a fluorescence plate reader at $440 \mathrm{~nm}$ with an excitation wavelength of $370 \mathrm{~nm}$.

Absorbance quantification of DNA-NP coverage with antigen. For functionalization of purified DNA-NPs with AF647-labelled peptide antigens, ratiometric absorbance measurements were used to quantify coverage. The concentration of DNA-NPs was determined via absorbance measurements at $260 \mathrm{~nm}$ using a NanoDrop 2000 spectrophotometer and compared to the concentration of AF647 determined at $647 \mathrm{~nm}\left(\varepsilon=270,000 \mathrm{~cm}^{-1} \mathrm{M}^{-1}\right)$. Coverage was determined in triplicate and absorbance values were extracted from the same UV-vis spectrum.

B-cell calcium flux assay. Ramos B cells at a concentration of 10 million cells per $\mathrm{ml}$ were incubated with $10 \mu \mathrm{M}$ Fluo- $4 \mathrm{AM}$ (ThermoFisher) for $30 \mathrm{~min}$ at $37^{\circ} \mathrm{C}$. After washing once, flux assays were performed on a Tecan plate reader at $37^{\circ} \mathrm{C}$ on a 96-well microplate with $160 \mu$ l of Fluo-4 labelled Ramos cells at 2 million cells per $\mathrm{ml}$. A baseline fluorescence was then recorded for $1 \mathrm{~min}$, and $40 \mu \mathrm{l}$ of NPs were added to the cells for a final concentration of $5 \mathrm{nM}$ of antigen, unless otherwise stated. A fixed concentration of antigens was used rather than the concentration of DNA-NPs to simplify comparison between experiments with various DNA-NPs and to assess the role of antigen concentration instead of the role of the DNA-NPs. For studies utilizing the $\mathrm{p} 5$ peptide antigen, primary B cells were isolated from 3-83 mouse spleens and stained via the same procedure. Primary B cells were isolated from splenocytes by negative selection using a StemCell EasySep B Cell Isolation Kit.

Animals. Female 3-83 mice (H-2K $\mathrm{K}^{\mathrm{K}}$-specific $\left.\mathrm{BCR}\right), 6-10$ weeks of age, were used for primary B cell experiments. Mice were handled under local, state and federal guidelines following an Institutional Animal Care and Use Committee (IACUC)-approved protocol at MIT.

B-cell calcium flux data statistical analysis. Raw calcium traces were normalized to a common baseline by subtracting the PBS time trace at every time point, then dividing the time trace at every point by the average of the time points before antigen addition. The time points after antigen addition were then summed for each sample in each repeat to give the calcium release above baseline $\left(I_{\text {tot }}\right)$. The maximum $I_{\text {tot }}$ across all samples within each repeat was determined $\left(\max \left(I_{\text {tot }}\right)\right)$, and the total calcium signalling (normalized AUC) for each sample within each repeat is then given by $I_{\mathrm{tot}} / \max \left(I_{\mathrm{tot}}\right)$. Repeats were averaged together to yield the bar height for the graphs in Figs. 2-4. Student's $t$-test was performed on the normalized AUCs entering into this average, where in most cases $N=3$ replicates.

B-cell imaging. Sample preparation for confocal microscopy. Ramos cells were labelled on ice at a concentration of 5 million per $\mathrm{ml}$ and protected from light for $30 \mathrm{~min}$ in Hank's buffered salt solution (HBSS) with $20 \mu \mathrm{g} \mathrm{ml}^{-1}$ human anti IgM $\mathrm{f}(\mathrm{Ab}) 1$ fragment (Jackson 109-007-043) conjugated to Janelia Fluor 549. Cells were spun down and resuspended in warm HBSS at a concentration of 2 million per $\mathrm{ml}$. Antigens were added to a final concentration of $5 \mathrm{nM}$ by adding $50 \mu \mathrm{l}$ antigen solution to a volume of cells between $175 \mu \mathrm{l}$ and $400 \mu \mathrm{l}$, and cells were kept at $37^{\circ} \mathrm{C}$ by incubation in a thermal bead bath. At time points following the addition of antigen, $100 \mu \mathrm{l}$ of cells were removed and placed into $200 \mu \mathrm{l}$ of $6 \%$ warm PFA solution and allowed to fix for $10 \mathrm{~min}$ at $37^{\circ} \mathrm{C}$. Following fixation, fixed cells were permeabilized by HBSS containing $0.1 \%$ Triton-X and washed before being diluted in $4.5 \mathrm{ml} \mathrm{HBSS}$ and centrifuged at $600 \mathrm{~g}$ for $5 \mathrm{~min}$. Cells were then labelled for $5 \mathrm{~h}$ at $4^{\circ} \mathrm{C}$ in $50 \mu \mathrm{l} \mathrm{HBSS}$ with a 1:100 dilution of anti-phospho Syk primary Ab (Millipore Sigma) and a 1:50 dilution of phalloidin conjugated to Alexa 405 in the presence of $5 \mathrm{mg} \mathrm{ml}^{-1}$ bovine serum albumin (BSA, Sigma) and $0.1 \%$ Triton-X. Cells were diluted into $4.5 \mathrm{ml} \mathrm{HBSS}$ and centrifuged at $600 \mathrm{~g}$ for $5 \mathrm{~min}$, then resuspended in $4.5 \mathrm{ml} \mathrm{HBSS}$ and centrifuged again to wash before being resuspended in $100 \mu \mathrm{l}$
HBSS. Cells were then labelled with a 1:100 dilution of secondary anti-rabbit conjugated to AF 488 for $1 \mathrm{~h}$ at $4^{\circ} \mathrm{C}$ in the presence of $5 \mathrm{mg} \mathrm{ml}^{-1} \mathrm{BSA}$ and $0.1 \%$ Triton-X, before being washed twice as above. Cells were then plated onto LabTech II eight-well glass-bottomed chambers modified with $0.1 \%$ poly-L-lysine (Sigma P8920) and allowed to adhere for at least $4 \mathrm{~h}$ at $4^{\circ} \mathrm{C}$ before performing confocal microscopy

Confocal microscopy imaging. Confocal microscopy was performed on a Zeiss AxioVert 200M inverted microscope stand with a Yokogawa CSU-22 spinning disk confocal scan head and an Andor Borealis multi-point confocal system. Probes were excited by four laser lines in the Andor/Spectral Applied Research Integrated Laser Engine: $405 \mathrm{~nm} 100 \mathrm{~mW}$ optically pumped semiconductor laser (OPSL), $488 \mathrm{~nm} 150 \mathrm{~mW}$ OPSL, $561 \mathrm{~nm} 100 \mathrm{~mW}$ OPSL and $642 \mathrm{~nm} 110 \mathrm{~mW}$ OPSL. Multipass dichroic mirror $(405 / 488 / 568 / 647 \mathrm{~nm})$ and emission filters $(450 / 50 \mathrm{~nm}$, $525 / 50 \mathrm{~nm}, 605 / 70 \mathrm{~nm}$ and $700 / 75 \mathrm{~nm}$ ) were used for each emission channel, respectively. Each sample was imaged through a $\times 63$ oil Plan Apochromat objective with an effective pixel size of $0.092 \mu \mathrm{m}$ per pixel. Images were captured through a Hamamatsu Orca-ER cooled charge-coupled device, and instrumentation was controlled through MetaMorph software. For each image, nine $z$-planes with separation of $1.5 \mu \mathrm{m}$ were acquired between the top and bottom of the cell, and $\sim 10$ fields of view were acquired for each sample.

Image analysis. Sixteen-bit images were read into MATLAB and converted to double precision. For each field of view, a maximum intensity projection (MIP) was calculated for the phalloidin channel. This was then binarized using adaptive thresholding, cleaned of stray pixels and then morphological opening and closing was performed. Holes within this binarization were then filled, and discrete objects within this binarization were labelled as individual cells. For each cell in a field of view, $z$-planes were binarized as above using the phalloidin channel, and these $z$-plane binarizations were restricted to the limit of the MIP binarization for each cell. The convex hull of this $z$-plane binarization was used to estimate the extent of the cell, and the cell surface was estimated by selecting the perimeter of the $z$-plane binarization and dilating this 25 times in a four-connected neighbourhood, then subsequent restriction by the undilated cell extent binarization. The total intensity of cellular probes and the surface intensity of cellular probes were calculated by summation using all $z$-stacks after logical indexing of the background-subtracted raw $z$-plane images, where background was estimated to be a constant through all $z$-planes and channels. Pixel-based correlation was performed through pairwise linear correlation of pixel values between channels following logical indexing. The shown average intensity values are an average over cells, and error bars show the s.e.m., given by the s.d. divided by $\operatorname{sqrt}\left(N_{\text {cells }}\right)$. The internalized fraction of probe intensity for a single cell is given by (total cell intensity - cell surface intensity)/total cell intensity.

Reporting Summary. Further information on research design is available in the Nature Research Reporting Summary linked to this Article.

\section{Data availability}

The raw data that support the plots within this paper are available from the authors upon reasonable request.

\section{Code availability}

Computer code is available from GitHub at https://github.com/jayajitdas/ bcr-signaling-model.

\section{References}

43. Weaver, G. C. et al. In vitro reconstitution of B cell receptor-antigen interactions to evaluate potential vaccine candidates. Nat. Protoc. 11, 193-213 (2016)

44. Villar, R. F. et al. Reconstituted B cell receptor signaling reveals carbohydrate-dependent mode of activation. Sci. Rep. 6, 36298 (2016).

45. Veneziano, R. et al. In vitro synthesis of gene-length single-stranded DNA. Sci. Rep. 8, 6548 (2018).

46. Shepherd, T. R., Du, R. R., Huang, H., Wamhoff, E.-C. \& Bathe, M. Bioproduction of pure, kilobase-scale single-stranded DNA. Sci. Rep. 9, 6121 (2019).

47. Havenar-Daughton, C. et al. Rapid germinal center and antibody responses in non-human primates after a single nanoparticle vaccine immunization. Cell Rep. 29, 1756-1766.e8 (2019).

48. Marras, A. E., Zhou, L., Su, H.-J. \& Castro, C. E. Programmable motion of DNA origami mechanisms. Proc. Natl Acad. Sci. USA 112, 713-718 (2015).

49. Schneider, C. A., Rasband, W. S. \& Eliceiri, K. W. NIH Image to ImageJ: 25 years of image analysis. Nat. Methods 9, 671-675 (2012).

\section{Acknowledgements}

This work was supported by the Human Frontier Science Program (RGP0029/2014), the Office of Naval Research (N00014-16-1-2953), the US Army Research Office 
through the Institute for Soldier Nanotechnologies at MIT (cooperative agreement no. W911NF-18-2-0048), the Ragon Institute of MGH, MIT and Harvard, and the NIH (R21-EB026008, R01-MH112694, AI048240, UM1AI144462 and UM1AI100663). W.R.S. acknowledges funding from the IAVI Neutralizing Antibody Consortium (NAC) and Center, and the Collaboration for AIDS Vaccine Discovery funding for the IAVI NAC Center. J.D. is supported by the NIH (R01-AI 143740, R01-AI 146581). E.-C.W. is supported by the Feodor Lynen Fellowship of the Alexander von Humboldt Foundation. D.J.I. is an investigator of the Howard Hughes Medical Institute.

\section{Author contributions}

R.V. designed the different DNA-NPs and performed folding and characterization of the DNA-NPs, conjugations with different antigens and their fluorescence quantification measurements, and analysed the data. T.J.M. performed antigen modification with PNA and fluorescent dye, the B-cell calcium flux assay, the flow cytometry and analysed the data. M.B.S. performed confocal microscopy imaging, image analysis and analysed the data. E.-C.W. performed additional DNA-NPs synthesis, modification with antigens and characterization. T.R.S. performed TEM characterization. B.J.R. performed additional antigen modification with PNA, B-cell calcium flux and data analysis. S.M. and J.D. developed the in silico model. S.M. developed computer codes, and S.M. and J.D. simulated the model and analysed the data. W.R.S. designed the immunogens. M.B. and D.J.I. designed and supervised the study and interpreted the results. R.V., T.J.M., M.B.S., J.D., D.J.I. and M.B. wrote the manuscript. All authors commented on and edited the manuscript.

\section{Competing interests}

The authors declare no competing interests.

\section{Additional information}

Supplementary information is available for this paper at https://doi.org/10.1038/ s41565-020-0719-0.

Correspondence and requests for materials should be addressed to D.J.I. or M.B.

Peer review information Nature Nanotechnology thanks Chunhai Fan, Michael Reth and the other, anonymous, reviewer(s) for their contribution to the peer review of this work.

Reprints and permissions information is available at www.nature.com/reprints. 


\section{natureresearch}

\section{Reporting Summary}

Nature Research wishes to improve the reproducibility of the work that we publish. This form provides structure for consistency and transparency in reporting. For further information on Nature Research policies, see Authors \& Referees and the Editorial Policy Checklist.

\section{Statistics}

For all statistical analyses, confirm that the following items are present in the figure legend, table legend, main text, or Methods section.

n/a Confirmed

$\square$ \ The exact sample size $(n)$ for each experimental group/condition, given as a discrete number and unit of measurement

$\square$ \A statement on whether measurements were taken from distinct samples or whether the same sample was measured repeatedly

The statistical test(s) used AND whether they are one- or two-sided

Only common tests should be described solely by name; describe more complex techniques in the Methods section.

$\square$ \ A description of all covariates tested

$\square$ \ A description of any assumptions or corrections, such as tests of normality and adjustment for multiple comparisons

$\square$ A full description of the statistical parameters including central tendency (e.g. means) or other basic estimates (e.g. regression coefficient)

AND variation (e.g. standard deviation) or associated estimates of uncertainty (e.g. confidence intervals)

$\varnothing$ For null hypothesis testing, the test statistic (e.g. $F, t, r$ ) with confidence intervals, effect sizes, degrees of freedom and $P$ value noted

Give P values as exact values whenever suitable.

Х $\square$ For Bayesian analysis, information on the choice of priors and Markov chain Monte Carlo settings

$\square$ \ For hierarchical and complex designs, identification of the appropriate level for tests and full reporting of outcomes

$\square$ Xstimates of effect sizes (e.g. Cohen's $d$, Pearson's $r$ ), indicating how they were calculated

Our web collection on statistics for biologists contains articles on many of the points above.

\section{Software and code}

Policy information about availability of computer code

Data collection Confocal imaging experiments: Metamorph 7.8. In silico model data: Model was coded using software package Stochastic Simulation Compiler, which is freely available from the link http://web.mit.edu/irc/ssc/. The specific codes are available with submission in ssc_code_param.zip file. We plan to make this available to the community via Github.

Data analysis

Custom software written in MATLAB 2018b was used for analysis. FlowJo (v.10.0.8) was used to analyze the flow cytometry data. ImageJ (v1.52a) was used to merge the fluorescent image of the agarose gels.

For manuscripts utilizing custom algorithms or software that are central to the research but not yet described in published literature, software must be made available to editors/reviewers. We strongly encourage code deposition in a community repository (e.g. GitHub). See the Nature Research guidelines for submitting code \& software for further information.

\section{Data}

Policy information about availability of data

All manuscripts must include a data availability statement. This statement should provide the following information, where applicable:

- Accession codes, unique identifiers, or web links for publicly available datasets

- A list of figures that have associated raw data

- A description of any restrictions on data availability

The data generated and analyzed that support the findings within this paper as well as the various DNA origami designs are available from the corresponding author upon reasonable request to the corresponding author. 


\section{Field-specific reporting}

Please select the one below that is the best fit for your research. If you are not sure, read the appropriate sections before making your selection. \Life sciences

$\square$ Behavioural \& social sciences Ecological, evolutionary \& environmental sciences

For a reference copy of the document with all sections, see nature.com/documents/nr-reporting-summary-flat.pdf

\section{Life sciences study design}

All studies must disclose on these points even when the disclosure is negative.

Sample size A sample size of 3 was used to detect a significant difference $(p<0.05)$ between groups with a signal to noise ratio of 3.0 with $80 \%$ power. Calcium assay: sample size is the number of unique wells from a single plate reader calcium assay. This was chosen by the maxiumum number of replicates in a sample that could be performed given limitations of the experiment. The variance between samples from individual experiments was deemed small enough to merit the sample sizes chosen.

Microscopy: These are the number of cells analyzed from a single preparation of a slide. These sample sizes were deemed acceptable given the observed variation in cell signals within each experiment.

Data exclusions Calcium data: runs were excluded where control stimulants (eOD60 and ionomycin) do not show any response from fluorescent probe Microscopy: No data were excluded

Replication

Experiments were performed multiple times to confirm observations. The number of replicates (biological or technical) for each experiments is provided in the caption of each figures. All attempts at replication were successful.

Randomization Cells used for test experiments were randomly assigned to experimental groups by the process of sample preparation. No other randomization was performed.

Blinding

Experimenters were blind to the type of nanoparticle used within the samples.

\section{Reporting for specific materials, systems and methods}

We require information from authors about some types of materials, experimental systems and methods used in many studies. Here, indicate whether each material, system or method listed is relevant to your study. If you are not sure if a list item applies to your research, read the appropriate section before selecting a response.

Materials \& experimental systems

Methods

$\mathrm{n} / \mathrm{a}$ Involved in the study

$\square$ \ Antibodies

$\square$ Eukaryotic cell lines

\ $\square$ Palaeontology

$\square \bigotimes$ Animals and other organisms

$\bigotimes \square$ Human research participants

$\bigotimes \square$ Clinical data

\begin{tabular}{l|l}
\hline n/a & Involved in the study \\
$\square$ & $\square$ ChIP-seq \\
$\square$ & $\bigotimes$ Flow cytometry \\
$\square$ & $\square$ MRI-based neuroimaging
\end{tabular}

\section{Antibodies}

Antibodies used

Validation
BCR probe: Goat Anti-Human anti IgM f(Ab)1 fragment (Jackson; Product number: 109-007-043), 1:100 dilution Anti-phospho Syk Ab (Millipore Sigma; Product number: PK1010), 1:100 dilution Phalloidin AlexaFluor 405 Ab (ThermoFisher; Product number: A30104), 1:50 dilution Goat anti-rabbit IgG AlexaFluor488 Ab (ThermoFisher; Product number: PK1010), 1:100 dilution

Anti-human IgM fAb1 fragment was confirmed to not bind non-lgM expressing cells. Manufacturer states that "based on antigen-binding assay and/or ELISA, the antibody reacts with the Fc5 $\mu$ portion of the human IgM heavy chain but not with human IgG, IgA, or the light chains of human immunoglobulins." (https://www.jacksonimmuno.com/catalog/ products/109-007-043).

Anti-phospho Syk Ab was only detected within Ramos B cells following addition of stimulatory antigens. Was not detected on cell surface if cells were not permeablized. 
Policy information about cell lines

Cell line source(s)

Ramos B cells expressing germline VRC01 were provided by Daniel Lingwood (Ragon Institute). Expi293 cells were obtained from ThermoFisher Scientific.

Authentication

Ramos B cells expressing germline VRCO1 were authenticated by measuring the binding of fluorescently labeled eOD probes. Expi293 cells were not validated.

Mycoplasma contamination

All cell lines tested negative for mycoplasm.

Commonly misidentified lines

(See ICLAC register)

No commonly misidentified lines were used in this study.

\section{Animals and other organisms}

Policy information about studies involving animals; ARRIVE guidelines recommended for reporting animal research

Laboratory animals

Female 3-83 mice, 6-10 weeks old were used in this study.

Wild animals

The study did not involve wild animals.

Field-collected samples

The study did not involve samples collected from the field.

Ethics oversight

Mice were handled under local, state, and federal guidelines following an Institutional Animal Care and Use Committee (IACUC)aproved protocol at MIT.

Note that full information on the approval of the study protocol must also be provided in the manuscript.

\section{Flow Cytometry}

Plots

Confirm that:

\The axis labels state the marker and fluorochrome used (e.g. CD4-FITC).

Х The axis scales are clearly visible. Include numbers along axes only for bottom left plot of group (a 'group' is an analysis of identical markers).

\All plots are contour plots with outliers or pseudocolor plots.

\A numerical value for number of cells or percentage (with statistics) is provided.

\section{Methodology}

Sample preparation

Ramos B cells expressing germline VRC01 were stained for 30 minutes with AlexaFluor647-lableled eOD $(5 \mathrm{nM})$ constructs on ice. Cells were washed in PBS 1\% BSA once, and cells were stained with DAPI as the live/dead stain.

Instrument

BD FACSCanto II HTS-1 was used for flow cytometry data collection.

Software

FlowJo Version 10.0.8 was used for data analysis.

Cell population abundance

At least 10,000 cells were analyzed for each condition.

Gating strategy

Cells were gated first by FSC/SSC, and then for DAPI negative (live cells).

Xick this box to confirm that a figure exemplifying the gating strategy is provided in the Supplementary Information. 